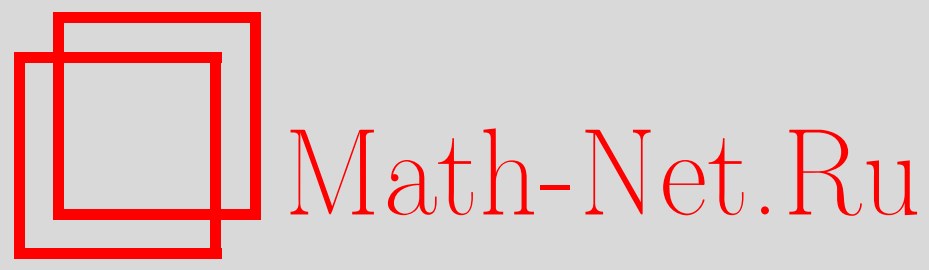

А. Г. Хованский, Об одной лемме Концевича, Функи. анализ и его прил., 1997, том 31, выпуск 4, 89-91

DOI: https://doi.org/10.4213/faa477

Использование Общероссийского математического портала MathNet.Ru подразумевает, что вы прочитали и согласны с пользовательским соглашением

http://www . mathnet.ru/rus/agreement

Параметры загрузки:

IP : 52.90 .164 .192

26 апреля 2023 г., 06:58:48

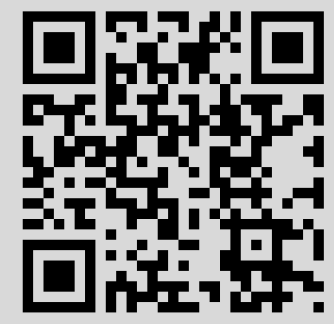




\title{
Об одной лемме Концевича
}

\author{
(c) 1997. А. Г. ХовАнский
}

В. И. Арнольду к его шестидесятилетию

Недавно М. Концевич построил универсальную теорию квантования. Среди открытых им фактов содержится следующее утверждение. Пусть $f_{1}, \ldots, f_{2 n}$ суть $2 n$ рациональных функций на полном комплексном алгебраическом многообразии $M$, комплексная размерность которого равна $n$. Пусть $M_{0}$ - множество неособых точек алгебраического многообразия $M$, из которого выброшено объединение носителей дивизоров функций $f_{1}, \ldots, f_{2 n}$. Обозначим через $\arg f_{i}$ аргумент функщии $f_{i}$.

ЛЕмма КонцеВичА. $\int_{M_{0}} d \arg f_{1} \wedge \cdots \wedge d \arg f_{2 n}=0$.

В последнем списке задач В. И. Арнольда есть следующая задача: дать наглядное доказательство леммы Конщевича. В настоящей заметке такое доказательство приводится.

\section{1. Преобразование дифференциальной формы.}

Лемма 1. Справедливо тождество

$$
d \arg f_{1} \wedge \cdots \wedge d \arg f_{2 n} \equiv d \ln \left|f_{1}\right| \wedge \cdots \wedge d \ln \left|f_{2 n}\right| .
$$

ДокАЗАТЕЛЬСТво. Пусть $I_{x}$ - оператор умножения на мнимую единицу в касательном пространстве к многообразию $M$ в неособой точке $x$. Мы будем рассматривать $I_{x}$ как вещественное линейное преобразование. Во-первых, определитель этого преобразования равен единице. Во-вторых, в силу аналитичности функции $\ln f_{i}$ справедливо тождество

$$
I_{x}^{*} d \arg f_{i}=d \ln \left|f_{i}\right| .
$$

Отсюда и вытекает лемма 1.

Функции $\ln \left|f_{i}\right|$ в отличие от функций $\arg f_{i}$ являются однозначными. Поэтому лемма 1 почти полностью объясняет лемму Концевича. Единственное оставшееся затруднение доставляют особенности функций $\ln \left|f_{i}\right|$. Покажем, как его преодолеть.

2. Разрешение особенностей. Рассмотрим рациональное отображение $F: M \rightarrow\left(\mathbb{C P}^{1}\right)^{2 n}$, переводящее точку $x$ в точку $\left(f_{1}(x), \ldots, f_{2 n}(x)\right)$. Согласно теореме Хиронаки, существуют неособое компактное комплексное многообразие $N$ и регулярное отображение $\pi: N \rightarrow M$, такие, что

1) общая точка многообразия $M$ имеет единственный прообраз при отображении $\pi$;

2) отображение $G=F \circ \pi$ регулярно;

* Работа выполнена при частичной поддержке гранта № 95-011-8701 Российского фонда фундаментальных исследований и Канадского гранта No. 0GP0156833. 
3) объединение $\Gamma$ носителей дивизоров функций и $g_{i}=f_{i} \circ \pi$ является объединением трансверсально пересекающихся гладких гиперповерхностей в многообразии $N$.

Лемму Концевича достаточно доказать для многообразия $N$ и функций $g_{1}, \ldots, g_{2 n}$. Действительно, многообразия $M$ и $N$ различаются лишь множеством меньшей размерности, и это различие никак не сказывается на интегралах.

3. Полярные координаты. Около точек гиперповерхности $Г$ на многообразии $N$ удобно ввести полярные координаты. Пусть $U-$ координатная окрестность на многообразии $N$ и $z_{1}, \ldots, z_{n}$ - такие координаты, что гиперповерхность $\Gamma$ в карте $U$ задается уравнением $z_{1} \cdots z_{k}=0$, где $k$ - некоторое неотрицательное число. Отождествим область $U$ с ее образом в $\mathbb{C}^{n}$ при вложении, заданном координатными функциями $z_{j}$. Пусть $\mathbb{R}^{2 n}$ - пространство с координатными функциями $r_{1}, \varphi_{1}, \ldots, r_{k}, \varphi_{k}, x_{k+1}, y_{k+1}, \ldots, x_{n}, y_{n}$ и $V \subseteq \mathbb{R}^{2 n}$ - область, определенная неравенствами $r_{1} \geqslant 0, \ldots, r_{k} \geqslant 0,0 \leqslant \varphi_{1} \leqslant 2 \pi, \ldots$, $0 \leqslant \varphi_{k} \leqslant 2 \pi$.

Рассмотрим отображение $\rho$ области $V$ в $\mathbb{C}^{n}$, заданное формулами $z_{j}=r_{j} e^{i \varphi_{j}}$ при $1 \leq j \leqslant k$ и $z_{j}=x_{j}+i y_{j}$ при $k<j \leqslant n$.

ЛЕмма 2. Форма $\rho^{*} d \ln \left|g_{1}\right| \wedge \cdots \wedge \rho^{*} d \ln \left|g_{2 n}\right|$ является гладкой в прообразе области $U$.

ДокАЗАТЕЛЬСтво. Пусть $g$ - одна из функций $g_{1}, \ldots, g_{2 n}$. По условию функция $g$ в области $U$ представима в виде произведения функции, не обращающейся в нуль, на моном $z_{1}^{m_{1}} \cdots z_{k}^{m_{k}}$. Поэтому $d \ln |g|=\sum m_{j} \frac{d r_{j}}{r_{j}}+\alpha$, где $\alpha-$ гладкая 1-форма в области $U$. Разложим гладкую 1-форму $\rho^{*} \alpha$ по координатному базису в области $V$. Коэффициент $A_{j}$ при $d \varphi_{j}$ в этом разложении делится на $r_{j}$, т.е. $A_{j}=r_{j} B_{j}$, где $B_{j}-$ гладкая функция. Поэтому в произведении $\rho^{*} d \ln \left|g_{1}\right| \wedge \cdots \wedge \rho^{*} \ln \left|g_{2 n}\right|$ отрицательные степени всех координатных функций $r_{j}$ сокращаются и полученная форма является гладкой.

СлЕдСТВИЕ. Интеграл из леммы Концевича абсолютно сходится.

После разрешения особенностей следствие вытекает из леммы 2. Действительно, компактное многообразие $N$ покрывается конечным числом карт $U$, в которых действует эта лемма.

4. Отображение степени нуль. Сопоставим комплексному числу его модуль. Это отображение продолжается по непрерывности до отображения комплексной проективной прямой в вещественную проективную прямую. Обозначим через $\mu:\left(\mathbb{C P}^{1}\right)^{2 n} \rightarrow\left(\mathbb{R P}^{1}\right)^{2 n}$ декартову степень этого отображения. Образ многообразия $\left(\mathbb{C P}^{1}\right)^{2 n}$ при отображении $\mu$ является замыканием в $\left(\mathbb{R P}^{1}\right)^{2 n}$ положительного октанта $\left(\mathbb{R}_{+}\right)^{2 n}$.

Пусть $G: N \rightarrow\left(\mathbb{C P}^{1}\right)^{2 n}, G=\left(g_{1}, \ldots, g_{2 n}\right)$ - регулярное отображение $n$-мерного многообразия $N$ в $\left(\mathbb{C P}^{1}\right)^{2 n}$ и $\Gamma$ - объединение носителей дивизоров Функций $g_{1}, \ldots, g_{2 n}$.

ЛЕмма 3. Орраничение на область $N \backslash \Gamma$ отображения $\mu \circ G$ является собственным отображением этой области во внутренность положительного октанта. Степень этого собственного отображения равна нулю. 
ДоКАЗАТЕЛЬСТВо. Прообраз гранищы положительного октанта в многообразии $\left(\mathbb{R P}^{1}\right)^{2 n}$ при отображении $\mu \circ G$ совпадает с гиперповерхностью Г. Поэтому ограничение этого отображения на область $N \backslash \Gamma$ является собственным отображением.

Рассмотрим на октанте $\left(\mathbb{R}_{+}\right)^{2 n}$ форму старшей степени

$$
\omega_{2 n}=\frac{d x_{1}}{x_{1}} \wedge \cdots \wedge \frac{d x_{2 n}}{x_{2 n}} .
$$

Объем октанта относительно такой формы бесконечен. Однако интеграл $\int_{N \backslash \Gamma}(\mu \circ G)^{*} \omega_{2 n}$ абсолютно сходится (см. следствие из п. 2). Поэтому почти каждая точка положительного октанта лежит вне образа области $N \backslash \Gamma$. Следовательно, степень отображения $\mu \circ G$ равна нулю.

5. Завершение доказательства. Согласно следствию из п. 2, интеграл из леммы Концевича абсолютно сходится. В силу леммы 3 этот интеграл равен нулю.

6. Замечание. После того как заметка была написана, мне удалось познакомиться с оригинальным доказательством М. Концевича (Kontsevich M. Deformation quantization of Poisson manifolds. Preliminary version). Оно основано на сходной идее. Но все детали различаются, и в нашем варианте они проще. 